\title{
Safety and Efficacy of a Preservative-Free Artificial Tear Containing Carboxymethylcellulose and Hyaluronic Acid for Dry Eye Disease: A Randomized, Controlled, Multicenter 3-Month Study
}

This article was published in the following Dove Press journal: Clinical Ophthalmology

\author{
Pasquale Aragona (D) \\ Jose M Benítez-del-Castillo ${ }^{2}$ \\ Minas T Coroneo (iD ${ }^{3}$ \\ Subhanjan Mukherji ${ }^{4}$ \\ Jacqueline Tan (1D) \\ Evelien Vandewalle ${ }^{6}$ \\ Algis Vingrys (D) ${ }^{7}$ \\ Haixia Liu $^{8}$ \\ Cindy Carlisle-Wilcox ${ }^{8}$ \\ Joseph Vehige ${ }^{8}$ \\ Peter A Simmons ${ }^{5,8}$ \\ 'Department of Biomedical Sciences, \\ Università di Messina, Messina, Italy; \\ ${ }^{2}$ Department of Ophthalmology, Hospital \\ Clinico de Madrid, Universidad \\ Complutense, Madrid, Spain; ${ }^{3}$ M.T. \\ Coroneo Pty. Ltd, Randwick, NSW, \\ Australia; ${ }^{4}$ Department of \\ Ophthalmology, James Paget University \\ Hospital, Great Yarmouth, UK; \\ ${ }^{5}$ Optometry and Vision Science, UNSW \\ Sydney, Sydney, NSW, Australia; \\ ${ }^{6}$ Department of Ophthalmology, \\ University Hospitals Leuven, Leuven, \\ Belgium; ' 7 niversity of Melbourne \\ EyeCare Clinic, Carlton, VIC, Australia; \\ ${ }^{8}$ Allergan, an AbbVie company, Irvine, \\ CA, USA
}

Correspondence: Peter A Simmons PCS Research, 166II Camille Place,

Yorba Linda, CA, USA

Tel + I-7|4-743-3352

Email pasimmons17@gmail.com
Purpose: To compare the efficacy and safety of an artificial tear combining the polymers carboxymethylcellulose (CMC) and hyaluronic acid (HA), to a formulation of CMC alone in subjects with dry eye.

Methods: A preservative-free artificial tear (CMC-HA) was compared with an existing artificial tear (CMC). Subjects with mild-to-severe signs and symptoms of dry eye were enrolled in this double-masked, randomized, multicenter trial, and dosed at least twice daily for 90 days, with follow-up visits at Days 7, 30, 60, and 90. Ocular Surface Disease Index (OSDI) was the primary outcome measure. Secondary outcome measures were tear break-up time (TBUT), ocular surface staining, Schirmer test with anesthesia, and visual analog scale (VAS) scores of dry eye symptom severity and formulation acceptability. Safety measures included adverse events, biomicroscopy, and visual acuity.

Results: A total of 460 subjects were enrolled across 45 sites (38 in Europe; 7 in Australia), of whom 454 were randomized to receive treatment. The per-protocol (PP) population consisted of 394 subjects, 364 (92.4\%) of whom completed the study. In the PP population, the mean \pm SD change from baseline in OSDI score at the primary timepoint, Day 90, was $-16.9 \pm 17.5$ for CMC-HA and $-16.0 \pm 16.1$ for CMC. CMC-HA was non-inferior to CMC based upon a confidence interval method. Both treatments significantly improved $(P<0.001)$ OSDI, symptom VAS scores, TBUT, and ocular surface staining from baseline at all followup visits, with minimal differences between groups. Greater reduction of overall ocular pain/ discomfort was reported in subjects using CMC-HA versus CMC $(P=0.048)$. Approximately $10 \%$ of subjects in each group reported treatment-related adverse events of generally mild to moderate severity.

Conclusion: The new CMC-HA formulation was effective and well tolerated, and demonstrates a greater potential for symptom relief compared with CMC. These data support implementation of this formula for the management of dry eye patients.

Keywords: carboxymethylcellulose, hyaluronic acid, artificial tears, dry eye

\section{Plain Language Summary}

Why Was the Study Done?

This study was conducted to determine the ability of a new artificial tear formulation, combining two water-retentive polymers, carboxymethylcellulose (CMC) $0.5 \%$ and hyaluronic acid (HA) $0.1 \%$, to relieve symptoms and signs of dry eye disease compared with a standard formulation containing $\mathrm{CMC} 0.5 \%$ alone. 


\section{What Did the Researchers Do and Find?}

Subjects with mild-to-severe signs and symptoms of dry eye received CMC-HA or $\mathrm{CMC}$ at least twice daily for 90 days, and changes in symptoms such as discomfort, grittiness, burning (as measured using the Ocular Surface Disease Index questionnaire) and signs (measured by tear break-up time, ocular surface staining, and the Schirmer test) were evaluated at Days 7, 30, 60, and 90 in the overall population, and subgroups of subjects defined by the type of dry eye (evaporative, aqueous-deficient, or mixed). Both treatments significantly improved symptoms and signs, but there were no treatment differences with the exception of a greater reduction in overall ocular pain/discomfort at Day 90 with CMC-HA. Significant reductions in symptoms and ocular staining with CMC-HA versus CMC alone were also observed at earlier timepoints in subjects with aqueous-deficient dry eye.

What Do These Results Mean?

Both artificial tear formulations are effective in the treatment of dry eye disease regardless of its cause, although there was evidence to suggest a potential benefit with CMC-HA versus CMC in subjects with aqueous-deficient dry eye. These results support the use of the CMC-HA formulation in a broad range of subjects with dry eye disease.

\section{Introduction}

Dry eye is a multifactorial disease of the ocular surface characterized by a loss of homeostasis of the tear film, and accompanied by ocular symptoms and signs. Tear film instability and hyperosmolarity, ocular surface inflammation and damage, and neurosensory abnormalities play etiological roles. ${ }^{1}$ The prevalence of dry eye increases with age, and symptoms are estimated to affect up to $50 \%$ of the global population. ${ }^{2}$ The prevalence of dry eye is expected to increase in the future due to longer life expectancy and patterns of activity that involve more intense visual tasks with computers, smart phones, and similar devices. ${ }^{2}$ Dry eye symptoms can significantly impact daily functions such as reading, driving, professional work, and social activities and diminish quality of life. ${ }^{3,4}$

Artificial tears are typically used for symptomatic treatment of mild to moderate dry eye, and are often combined with pharmacological agents or surgical procedures for moderate to severe disease. ${ }^{5,6}$ The primary role of artificial tears is to supplement the deficient tear film, which is critical for ocular comfort and optimal visual function. Novel artificial lubricants with different polymers are still being developed, with improvements in understanding of the role that an unstable and hyperosmolar tear film plays in ocular inflammation and dry eye disease (DED)..$^{7-10}$ The restoration of tear film stability is achieved using polymers that exhibit humectant and viscoelastic properties, but is also further enhanced through the use of osmoprotectants (or osmolytes). Osmoprotectants are naturally occurring compatible solutes that are internalized by the cell and help prevent cell volume loss, oxidative stress, and inflammation. ${ }^{11-14}$ Osmolytes include polyols (eg, erythritol, glycerin, sorbitol), methylamines (eg, betaine, glycine), certain amino acids (eg, L-carnitine, taurine), ${ }^{15}$ and a derivative of the amino acid L-glutamate, 5-oxo-2-pyrrolidinecarboxylic acid, which also displays high water binding capacity. ${ }^{16}$ Carboxymethylcellulose (CMC), a polymeric derivative of 5-oxo-2-pyrrolidinecarboxylic acid, and hyaluronic acid (HA), are water-retentive polymers that have been shown to act synergistically with osmoprotectants to improve symptoms and signs of dry eye. ${ }^{17}$

A new preservative-free artificial tear combining CMC $0.5 \%$ and HA $0.1 \%$ (Optive Fusion ${ }^{\circledR}$ unit dose [UD], Allergan, an AbbVie company, North Chicago, Illinois) was recently introduced for the treatment of dry eye. ${ }^{18}$ Since CMC and HA polymers readily adsorb to ocular surface cells via specific receptors, ${ }^{19,20}$ the new formulation was designed to stabilize the tear film, reduce corneal staining, and effectively lubricate and protect the ocular surface. Combining CMC and HA in a single formulation has previously demonstrated potential synergistic effects compared with individual CMC and HA solutions in terms of increased low-shear viscosity (characteristic of the tear film between blinks) while high-shear viscosity (the condition during blinking) was unaffected. ${ }^{21}$

In this study, the efficacy and safety of a multi-polymer artificial tear formulation containing CMC and HA were compared with a preservative-free formulation containing CMC alone in subjects with signs and symptoms of DED. Both formulations contain non-electrolyte organic osmolytes that function as osmoprotectants. ${ }^{13,22,23}$

\section{Study Methods}

\section{Trial Design}

This multicenter, randomized, double-masked, two-arm, parallel-group, 3-month study compared the efficacy and safety of a preservative-free artificial tear formulation containing CMC 0.5\% + HA 0.1\% (Optive Fusion UD Preservative Free Lubricant Eye Drops, Allergan, an AbbVie company) with a marketed preservative-free formulation containing CMC $0.5 \%$ alone (Refresh Optive ${ }^{\circledR}$ UD Preservative Free Lubricant Eye Drops, Allergan, an AbbVie company) in 
subjects with signs and symptoms of dry eye (ClinicalTrial. gov identifier: NCT01664949; EudraCT number: 2012 002238-35). The study was conducted at 38 centers in Europe (in Belgium, France, Germany, Italy, Russia, Spain, and the United Kingdom) and 7 centers in Australia between February 2013 and May 2014. The study conformed to the International Conference on Harmonisation guidelines for Good Clinical Practice. Investigators at each site obtained study approval from an independent ethics committee or institutional review board (The University of New South Wales, Human Research Ethics Committee [Australia]; Clinical Research Ethics Committee-University of Waterloo, Waterloo, Ontario, Research Ethics Review Committee and Health Research Ethics Board of Alberta Clinical Trials Committee, Edmonton, Alberta, Health Research Ethics Authority, St. John's Newfoundland and Labrador, Ottawa Health Science Network-Research Ethics Board, Ottawa, Ontario [Canada]); and (Schulman Associates Institutional Review Board, Inc [USA]), and all subjects provided written informed consent prior to the start of any study procedures.

\section{Key Inclusion and Exclusion Criteria}

The study enrolled male or female adult subjects who had an Ocular Surface Disease Index ${ }^{\circledR}$ (OSDI) score $\geq 18$ and $\leq 65$ (based on a scale of 0 to 100) and used artificial tears for dry eye at any time prior to study entry. Subjects using cyclosporine ophthalmic emulsion (Restasis ${ }^{\circledR}$, Allergan, an AbbVie company) were eligible provided the drops had been used for $\geq 6$ months prior to baseline. Subjects must have had three consecutive tear break-up time (TBUT) test scores $\leq 10$ seconds in at least one eye, and corneal and/or conjunctival staining of grade 1 to 4 (Oxford Scheme score range: 0 to 5) in at least one area that was related to dry eye in at least one eye.

Subjects were excluded if they had a Schirmer test (with anesthesia) score $\leq 2 \mathrm{~mm} / 5 \mathrm{~min}$ in either eye or corneal or conjunctival staining of grade 5 (Oxford Scheme) in any one area of either eye. Current contact lens wearers and subjects with recent changes to systemic medications affecting dry eye or vision were also excluded, as were subjects with recent ocular surface surgery.

\section{Study Treatment}

The study consisted of five visits, a baseline visit (Day 1) and four follow-up visits at Days 7, 30, 60, and 90 (or early exit). At baseline, subjects were
Table I Artificial Tears Evaluated in the Study

\begin{tabular}{|c|c|c|}
\hline Formulation $^{\mathbf{a}}$ & CMC ${ }^{b}$-HA UD & CMC UD \\
\hline $\begin{array}{l}\text { Brand name } \\
\text { (United States/ } \\
\text { Europe) }\end{array}$ & Optive Fusion $®$ UD & $\begin{array}{l}\text { Refresh Optive }{ }^{\circledR} \\
\text { Sensitive/Optive }{ }^{\mathrm{TM}} \text { UD } \\
\text { and Optava }{ }^{\mathrm{TM}}\end{array}$ \\
\hline Dosing & \multicolumn{2}{|l|}{ Unit-dose } \\
\hline Composition & $\begin{array}{l}\text { CMC, }{ }^{b} \text { H, glycerin, } \\
\text { sodium lactate, } \\
\text { levocarnitine, erythritol, } \\
\text { potassium chloride, } \\
\text { calcium chloride, } \\
\text { magnesium chloride, } \\
\text { purified water }\end{array}$ & $\begin{array}{l}\text { CMC, ' glycerin, boric } \\
\text { acid, sodium borate, } \\
\text { sodium citrate, } \\
\text { levocarnitine, } \\
\text { erythritol, potassium } \\
\text { chloride, calcium } \\
\text { chloride, magnesium } \\
\text { chloride, sodium } \\
\text { hydroxide, purified } \\
\text { water }\end{array}$ \\
\hline Viscosity $^{d}$ & $15.0 \mathrm{cP}$ & $14.8 \mathrm{cP}$ \\
\hline
\end{tabular}

Notes: ${ }^{\mathrm{a} F o r m u l a t i o n s ~ w e r e ~ i s o t o n i c ~ w i t h ~ a ~ n e u t r a l ~} \mathrm{pH}$; manufactured by Allergan (an AbbVie company), Dublin, Ireland; bow molecular weight ( $<100 \mathrm{kDa}$ ) CMC; ${ }^{\mathrm{C}}$ Mix of medium ( $\left.250 \mathrm{kDa}\right)$ and high molecular weight ( $\left.700 \mathrm{kDa}\right) \mathrm{CMC}$; ${ }^{\mathrm{d}}$ Standard Brookfield viscosity.

Abbreviations: CMC, carboxymethylcellulose; HA, hyaluronic acid; UD, unit dose.

randomized 1:1 to treatment with $\mathrm{CMC}-\mathrm{HA} \mathrm{UD}$ or CMC UD (Table 1) according to a randomization scheme prepared by Allergan Biostatistics. For patients who were using artificial tears at baseline, a washout period was not required. Assignments were performed via an automated interactive voice or web response system. At each site, subjects were stratified at baseline according to OSDI score into mild/moderate (18 to 32) and severe ( $>32$ to 65 ) dry eye subgroups, ${ }^{18}$ and according to a combined corneal/conjunctival staining score (sum score of three zones [nasal and temporal conjunctiva, and cornea], each zone scored from 0 to 5 based on a modified Oxford Scheme) into mild (1 to 3) and moderate/severe (4 to 12) surface damage subgroups. Subjects with a baseline Oxford Scheme staining sum score of 13 to 15 were excluded, as these scores would require at least one zone to have a score of 5 . Subjects received study eye drops at baseline and Days 30 and 60 visits and were instructed to instill one to two drops of the assigned study product into each eye, as needed, at least two times daily for 3 months. To maintain subject and investigator masking, eye drops were provided in identical 0.4-mL unit dose (UD) vials.

Subjects using topical intraocular pressure (IOP)-lowering medication and cyclosporine ophthalmic emulsion were 
allowed to continue at the same dose and frequency for the duration of the study.

\section{Outcome Measures}

The primary efficacy variable was the change in the mean OSDI score from baseline at Day $90 .{ }^{24}$ Since higher OSDI scores represent more severe disease, a negative change from baseline demonstrates improvement.

Secondary efficacy variables included the change from baseline in TBUT, corneal and conjunctival staining, and anesthetized Schirmer test at each visit. TBUT was obtained by the mean of 3 consecutive measurements in each eye following instillation of sodium fluorescein. Corneal staining was then assessed in each eye using a slit lamp with cobalt blue illumination and a yellow filter to enhance contrast, and graded on a scale of 0 (no staining) to 5 (severe staining) using the Oxford Scheme. ${ }^{25}$ Conjunctival staining with lissamine green was performed immediately after assessing corneal staining, and was also graded on a scale of 0 to 5 using the modified Oxford Scheme. Schirmer test with anesthesia was performed in each eye after completion of all other dry eye assessments. ${ }^{26}$ At each follow-up visit,

Table 2 Schedule of Visits and Procedures

\begin{tabular}{|c|c|c|c|c|c|}
\hline Study Day (Visit Window) & $\begin{array}{l}\text { I } \\
\text { (Baseline) }\end{array}$ & $\begin{array}{l}7 \\
( \pm 3 \text { Days })\end{array}$ & $\begin{array}{l}30 \\
( \pm 7 \text { Days })\end{array}$ & $\begin{array}{l}60 \\
( \pm 7 \text { Days })\end{array}$ & $\begin{array}{l}\text { 90/Early Exit } \\
\text { ( } 7 \text { Days) }\end{array}$ \\
\hline Written informed consent & $\mathbf{x}$ & & & & \\
\hline Inclusion/exclusion criteria assessment & $\mathbf{x}$ & & & & \\
\hline Demographics & $\mathbf{x}$ & & & & \\
\hline $\begin{array}{l}\text { Self-assessed paper source document questionnaires: } \\
\text { OSDI questionnaire } \\
\text { Symptom Survey } \\
\text { Study Eye Drop Experience Survey } \\
\text { Study Product Usage Questionnaire }\end{array}$ & $\begin{array}{l}\mathbf{x} \\
\mathbf{x}\end{array}$ & $\begin{array}{l}\mathbf{x} \\
\mathbf{x} \\
\mathbf{x} \\
\mathbf{x}\end{array}$ & $\begin{array}{l}\mathbf{x} \\
\mathbf{x} \\
\mathbf{x} \\
\mathbf{x}\end{array}$ & $\begin{array}{l}\mathbf{x} \\
\mathbf{x} \\
\mathbf{x} \\
\mathbf{x}\end{array}$ & $\begin{array}{l}\mathbf{x} \\
\mathbf{x} \\
\mathbf{x} \\
\mathbf{x}\end{array}$ \\
\hline Medical and ophthalmic histories & $\mathbf{x}$ & & & & \\
\hline Adverse event assessment & $\mathbf{x}$ & $\mathbf{x}$ & $\mathbf{x}$ & $\mathbf{x}$ & $\mathbf{x}$ \\
\hline Prestudy or concomitant medication assessment & $\mathbf{x}$ & $\mathbf{x}$ & $\mathbf{x}$ & $\mathbf{x}$ & $\mathbf{x}$ \\
\hline Concurrent procedures & & $\mathbf{x}$ & $\mathbf{x}$ & $\mathbf{x}$ & $\mathbf{x}$ \\
\hline Urine pregnancy test (for females of childbearing potential only) & $\mathbf{x}$ & & & & $\mathbf{x}$ \\
\hline Currently corrected distance visual acuity & $\mathbf{x}$ & $\mathbf{x}$ & $\mathbf{x}$ & $\mathbf{x}$ & $\mathbf{x}$ \\
\hline Best-corrected visual acuity & $\mathbf{x}$ & $\mathbf{x}$ & $\mathbf{x}$ & $\mathbf{x}$ & $\mathbf{x}$ \\
\hline Biomicroscopy & $\mathbf{x}$ & $\mathbf{X}$ & $\mathbf{X}$ & $\mathbf{X}$ & $\mathbf{x}$ \\
\hline Tear break-up time (with fluorescein) & $\mathbf{x}$ & $\mathbf{x}$ & $\mathbf{x}$ & $\mathbf{x}$ & $\mathbf{x}$ \\
\hline $\begin{array}{l}\text { Corneal staining (Oxford Scheme, with } \\
\text { fluorescein) }\end{array}$ & $\mathbf{x}$ & $\mathbf{x}$ & $\mathbf{x}$ & $\mathbf{x}$ & $\mathbf{x}$ \\
\hline Conjunctival staining (Oxford Scheme, with lissamine green) & $\mathbf{x}$ & $\mathbf{x}$ & $\mathbf{X}$ & $\mathbf{x}$ & $\mathbf{x}$ \\
\hline Schirmer test (with anesthesia) & $\mathbf{x}$ & $\mathbf{x}$ & $\mathbf{x}$ & $\mathbf{x}$ & $\mathbf{x}$ \\
\hline Intraocular pressure (with anesthesia and fluorescein) & $\mathbf{x}$ & $\mathbf{x}$ & $\mathbf{x}$ & $\mathbf{x}$ & $\mathbf{x}$ \\
\hline Study product dispensed & $\mathbf{x}$ & & $\mathbf{x}$ & $\mathbf{x}$ & \\
\hline Study product returned & & & $\mathbf{x}$ & $\mathbf{x}$ & $\mathbf{x}$ \\
\hline Product accountability & & (X) & $\mathbf{x}$ & $\mathbf{x}$ & $\mathbf{x}$ \\
\hline
\end{tabular}

Abbreviations: OSDI, Ocular Surface Disease Index; $X$, required; $(X)$, only if needed. 
subject perception of individual dry eye symptoms was assessed using the Symptom Survey (burning/stinging, grittiness/foreign body sensation, dryness, difficult/uncomfortable vision, and ocular pain/discomfort) based on a visual analog scale (VAS; 0 [no symptoms] to 100 [worst discomfort ever felt]), and short- and long-term drop use experience was assessed using a six-item (comfort/burning or stinging/ degree of blurring/normality of vision/quality of vision/longlasting relief of discomfort) VAS (0 [absence of symptoms] to 100 [worst discomfort ever felt]). Subjects were also asked to report their daily dosing frequency over the previous week.

Safety assessments included adverse events (AEs), undilated biomicroscopy, monocular currently corrected distance visual acuity and best-corrected distance visual acuity, and IOP at each visit. The schedule of visits and procedures is summarized in Table 2.

\section{Data Analysis}

The clinical efficacy hypothesis was that the effect of a CMC and HA on the ocular surface was not inferior to that of CMC alone as measured by the change in OSDI score from baseline. The primary efficacy analysis of the change from baseline in OSDI score at day 90 was performed using an analysis of variance (ANOVA) model, with treatment and stratification factors of baseline OSDI score and baseline staining score as fixed effects. Analysis was performed on the perprotocol (PP) population (randomized subjects with no significant protocol violations) using combined non-inferiority and superiority tests. ${ }^{27}$ If the upper limit of the two-sided 95\% CI for the least-squares mean difference in OSDI response (change from baseline in OSDI score at Day 90) between the two treatments (CMC-HA UD minus CMC UD) was less than 4.7 units, the CMC-HA UD formulation was considered non-inferior to CMC UD. A two-sided $t$-test for superiority was then performed at the 5\% level if the CMCHA UD formulation was shown to be non-inferior to CMC UD. A sensitivity analysis was performed in the intent-totreat (ITT) population using the last-observation-carriedforward method and ANOVA model similar to the PP population above. Subgroup analyses of the primary efficacy variable were performed based on baseline OSDI score stratum (mild/moderate vs severe symptoms) and baseline combined corneal and conjunctival staining (mild vs moderate/ severe) using a two-way ANOVA model with treatment and baseline staining score as the main effect for the baseline OSDI subgroup analysis, and treatment and baseline OSDI as the main effect for the baseline combined staining subgroup analysis, in the PP and ITT populations.
A post hoc analysis of the primary and secondary efficacy variables was performed by subtype of DED at baseline in the PP population. According to the literature and recent guidelines, Schirmer scores (with anesthesia) $\leq 10 \mathrm{~mm} / 5 \mathrm{~min}$ and TBUT $<10$ seconds are considered abnormal and indicative of aqueous-deficient and evaporative dry eye, respectively. ${ }^{25,28}$ For the post hoc analysis, lower cut-offs for the Schirmer score $(5 \mathrm{~mm} / 5 \mathrm{~min}$ and $7 \mathrm{~mm} / 5 \mathrm{~min}$ ) and TBUT (7 seconds), respectively, were selected to be consistent with previous dry eye trials and to increase diagnostic specificity. $^{28-32}$ Three DED subtypes were identified: evaporative dry eye (Schirmer [with anesthesia] score $\geq 10 \mathrm{~mm} /$ 5 min and TBUT $<7$ seconds), aqueous-deficient dry eye (Schirmer score $<5 \mathrm{~mm} / 5 \mathrm{~min}$, TBUT $\geq 7$ seconds), and mixed dry eye (Schirmer score $<7 \mathrm{~mm} / 5 \mathrm{~min}$, TBUT $<7$ seconds). Subjects who did not meet the criteria for the DED subtype groups were excluded from the post hoc analysis.

Secondary efficacy variables were analyzed using ANOVA models for between-group differences based on the least-square means and Type III sum of squares constructed from the ANOVA model or paired $t$-tests for within-group differences. Categorical variables were summarized by frequency and percentage, and were analyzed using Pearson's Chi-square test or Fisher's exact test. Statistical significance was set at a level of $P<0.05$.

Based on a one-sided Type I error rate of 0.025 , and the assumptions of no inherent treatment difference and a common standard deviation of 15.65, approximately 176 subjects in each treatment group (352 subjects total) were required to complete the study in the PP population to obtain $80 \%$ power for a one-sided non-inferiority test for a between-group difference of 4.7 units in mean change from baseline in OSDI score at Day 90. Sample size and power calculations were conducted using procedure MTE0 of the nQuery Advisor + nTerim software, version 6.01 (Statistical Solutions, Boston, MA, USA). With a 1:1 treatment allocation and 13\% combined dropout and protocol deviation rate, a total of 405 subjects were to be enrolled in order to have 352 completed subjects in the PP population through day 90 .

\section{Results Study Subjects}

Of the 460 subjects enrolled, a total of 454 were randomized to receive treatment (222 subjects to CMC-HA UD, 232 subjects to CMC UD; ITT population); the PP population consisted of 394 subjects, 364 (92.4\%) of whom completed 


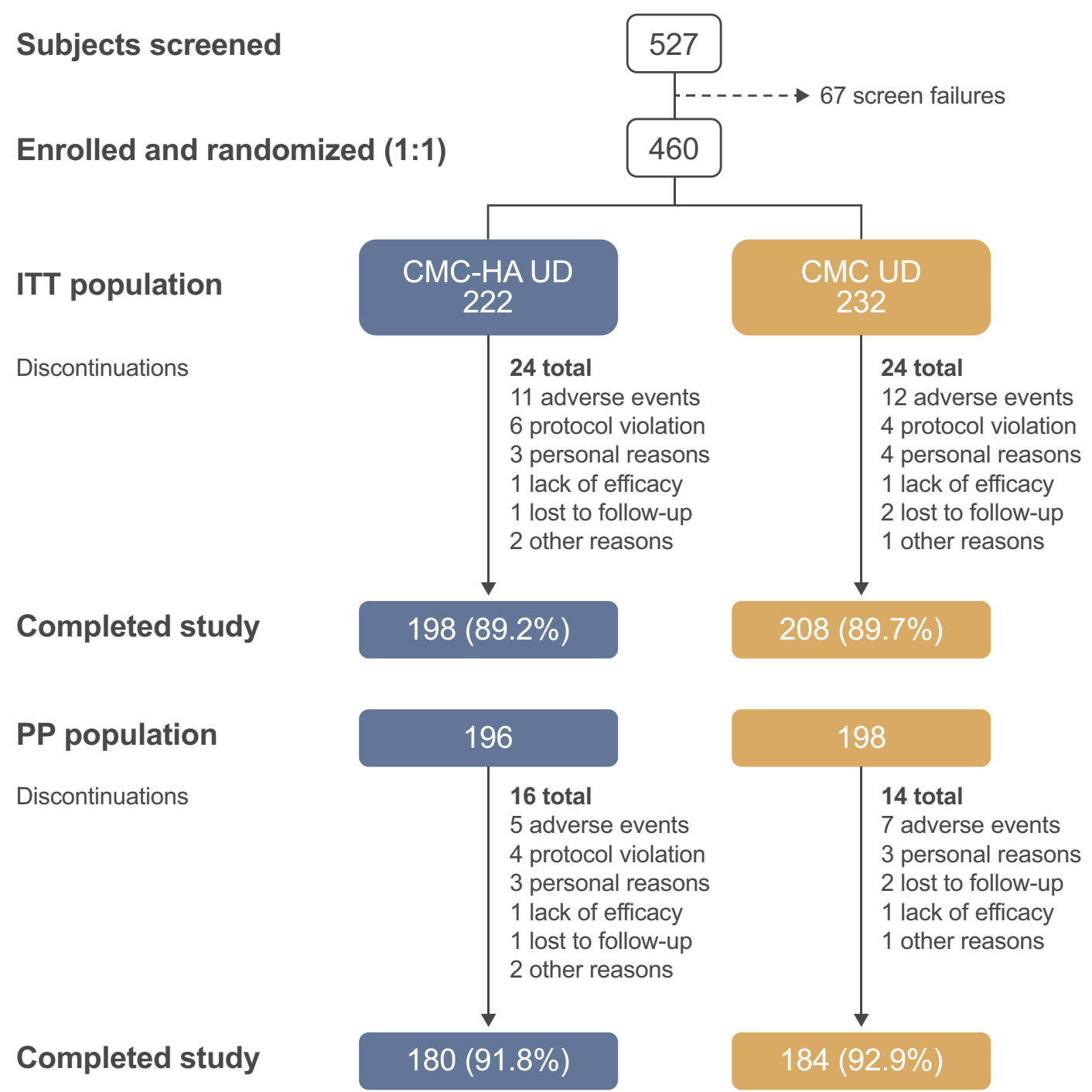

Figure I Subject flow through the study.

Abbreviations: ITT, intent-to-treat; PP, per-protocol; CMC, carboxymethylcellulose; HA, hyaluronic acid; UD, unit dose.

the study (Figure 1). The mean (standard deviation, SD) age of subjects in the ITT population was 58.4 (13.8) years and the majority (405 [89.2\%]) were older than 40 years of age. There were no significant differences in baseline characteristics and dry eye clinical assessments between treatment groups (Table 3). The most common ophthalmic conditions other than dry eye were cataract (19.4\%), blepharitis $(15.4 \%)$, and pinguecula (13.7\%). Before entering the study, subjects used a variety of ocular lubricants, including those containing hyaluronate sodium, polyethylene glycol 400/propylene glycol, carmellose, carbomer, or hypromellose for the treatment of dry eye. A history of topical cyclosporine use was reported by two patients in each group but the cyclosporine had been discontinued by one patient in each group before study screening.

\section{Primary Efficacy Variable}

In the PP population, no significant differences were observed between CMC-HA UD and CMC UD treatment groups in mean OSDI score change from baseline at any follow-up visit (Figure 2A). At Day 90, the mean (SD) change in OSDI score from baseline was -16.9 (17.5) for CMC-HA UD and -16.0 (16.1) for CMC UD. The leastsquares mean difference between the treatment groups was -0.75 and the $95 \%$ CI was -4.1 to 2.6 ; the upper limit of the $95 \% \mathrm{CI}$ was below the pre-specified clinical margin of 4.7, demonstrating that CMC-HA UD was non-inferior to CMC UD. Sensitivity analysis in the ITT population showed similar results and confirmed the non-inferiority of CMC-HA UD to CMC UD. Significant improvements in OSDI score from baseline were observed within both 
Table 3 Subject Baseline Characteristics (Intent-to-Treat Population)

\begin{tabular}{|c|c|c|}
\hline Characteristic & $\begin{array}{l}\text { CMC-HA UD } \\
(n=222)\end{array}$ & $\begin{array}{l}\text { CMC UD } \\
(n=232)\end{array}$ \\
\hline Mean (SD) age, years & $59.4(13.8)$ & $57.5(13.7)$ \\
\hline $\begin{array}{c}\text { Sex, n (\%) } \\
\text { Female } \\
\text { Male }\end{array}$ & $\begin{array}{l}180(81.1) \\
42(18.9)\end{array}$ & $\begin{array}{l}186(80.2) \\
46(19.8)\end{array}$ \\
\hline $\begin{array}{l}\text { Race, n (\%) } \\
\text { White } \\
\text { Non-white }\end{array}$ & $\begin{array}{l}194(87.4) \\
28(12.6)\end{array}$ & $\begin{array}{l}211(90.9) \\
21(9.1)\end{array}$ \\
\hline $\begin{array}{l}\text { Mean overall OSDI score } \\
(\mathrm{SD}) \\
\text { Mean TBUT (SD), sec } \\
\text { Mean Schirmer test } \\
(\mathrm{SD}), \mathrm{mm} / 5 \mathrm{~min}\end{array}$ & $\begin{array}{l}41.6(14.2) \\
4.4(1.9) \\
9.1(6.6)\end{array}$ & $\begin{array}{l}40.8(13.8) \\
4.4(1.9) \\
8.0(5.7)\end{array}$ \\
\hline $\begin{array}{l}\text { Mean staining score (SD) } \\
\text { Combined corneal/ } \\
\text { conjunctival } \\
\text { Corneal } \\
\text { Conjunctival }\end{array}$ & $\begin{array}{l}4.3(2.3) \\
1.5(1.0) \\
2.8(1.7)\end{array}$ & $\begin{array}{l}1.7(1.0) \\
3.0(1.8)\end{array}$ \\
\hline
\end{tabular}

Abbreviations: CMC, carboxymethylcellulose; HA, hyaluronic acid; $n$, number of subjects; OSDI, Ocular Surface Disease Index®; SD, standard deviation; TBUT, tear break-up time; UD, unit dose.

the CMC-HA UD and CMC UD treatment groups at Days 7, 30, 60, and $90(P<0.001)$, in both the PP and ITT populations. In addition, OSDI scores significantly improved in all three subscales (ocular symptoms, visionrelated functions, and environmental triggers) within both treatment groups at all study visits $(P<0.001)$.

Subgroup analysis based on baseline OSDI score revealed no significant differences between CMC-HA
UD and CMC UD in the mean change from baseline in OSDI score at any follow-up visit for the mild/moderate symptoms or the severe symptoms subgroups, in both the PP and ITT populations (Figure 2B). Similarly, no differences were observed between the two treatment groups for mean change from baseline in OSDI score at any followup visit for subjects in the mild combined staining subgroup or the moderate/severe combined staining subgroup, in both the PP and ITT populations (data not shown).

\section{Secondary Efficacy Variables}

Within-group mean changes from baseline in TBUT, combined corneal/conjunctival staining, corneal staining, and conjunctival staining scores were statistically significant at all follow-up visits in the CMC-HA UD and CMC UD treatment groups $(P<0.001)$ in both PP and ITT populations; however, there were no statistically significant between-group differences. In the PP population, mean changes from baseline in Schirmer test score were statistically significant in both treatment groups at Days 30, 60, and $90(P \leq 0.033)$, and in the CMC UD group at Day $7(P<0.001)$. At Day 90, the mean (SD) change was 1.4 (6.9) and 1.5 (6.3) in the CMC-HA UD and CMC UD groups, respectively. There were no significant between-group differences at any follow-up visit.

Mean changes from baseline in individual symptom scores in the Symptom Survey were statistically significant $(P<0.002)$ at each follow-up visit in both treatment groups in the PP and ITT populations (Figure 3A-E). At Day 90, the reduction in symptom scores was numerically greater with CMC-HA UD compared with CMC UD for all 5 VAS categories and was statistically significant in favor of

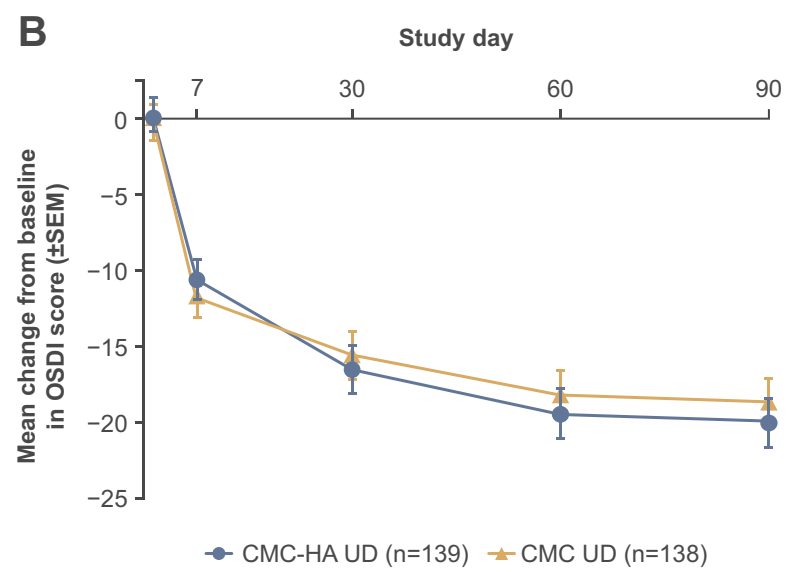

Figure 2 Mean change in Ocular Surface Disease Index (OSDI) score from baseline in (A) all subjects and (B) subjects with severe dry eye (OSDI score $>32)$ at baseline and at all study visits (per-protocol population). Between-group differences were not significant $(P>0.05)$.

Abbreviations: CMC, carboxymethylcellulose; HA, hyaluronic acid; SEM, standard error of mean; UD, unit dose. 
CMC-HA UD for overall ocular pain/discomfort in the PP population $(P=0.048$; Figure $3 \mathrm{E})$.

Overall, the average response to the Study Eye Drop Experience Survey questions concerning short- and longterm subjective experience with the study eye drops were comparable between CMC-HA UD and CMC UD at Day 90. VAS scores were $>70$ for each item, suggesting a high degree of comfort, effectiveness, quality of vision, and symptom improvement. In the PP population, subjects reported using CMC-HA UD and CMC UD eye drops a mean of 4.4 and 4.3 times per day, respectively, during the week preceding the final follow-up visit.

\section{Post Hoc Analysis}

After 90 days of CMC-HA UD or CMC UD treatment, improvements in dry eye symptoms (OSDI and VAS) and signs (ocular staining, TBUT, Schirmer test) were of similar magnitude in subjects with evaporative $(n=149)$ and mixed $(n=186)$ dry eye. In contrast, there was a clear trend in favor of CMC-HA UD for improving symptoms, TBUT, and Schirmer scores in the smaller cohort of aqueous-deficient subjects $(n=20)$. However, none of the between-group differences at Day 90 were statistically significant in any of the dry eye cohorts. In the respective evaporative, aqueousdeficient, and mixed dry eye cohorts, comparing CMCHA UD with CMC UD, the mean (SD) changes from baseline were as follows: OSDI scores $(-15.04$ [18.48] vs -12.46 [20.24], -19.53 [21.36] vs -17.16 [8.46]), -12.02 [23.06] vs -13.42 [18.31]); TBUT (2.51 seconds [3.61] vs 2.42 seconds [3.09], 4.31 seconds [12.10] vs 0.39 seconds [3.49], 1.54 seconds [4.79] vs 1.66 seconds [2.32]); Schirmer scores $(-0.57$ [6.70] vs -1.32 [7.31], 4.20 [5.15] vs 1.90 [4.15], 2.11 [6.50] vs 2.41 [5.36]). Treatment differences at Day 90 between CMC-HA UD and CMC UD in dry eye symptoms (VAS) were most notable in the aqueousdeficient cohort for the categories of burning/stinging ( -33.0 [30.8] vs -15.0 [20.8]), dryness ( -37.4 [30.6] vs -20.3 [35.3]), difficult/uncomfortable vision (-25.0 [25.0] vs -10.0 [16.6]) and overall ocular pain/discomfort $(-25.2$ [26.9] vs -6.0 [28.7]). Statistically significant betweengroup differences in favor of CMC-HA UD were evident at the earlier time points for the OSDI score at Day 7 in the evaporative dry eye cohort $(P=0.041)$, the overall ocular discomfort/pain $(P=0.009)$ and difficult/uncomfortable vision $(P=0.017)$ on Day 30 in the aqueous-deficient cohort,
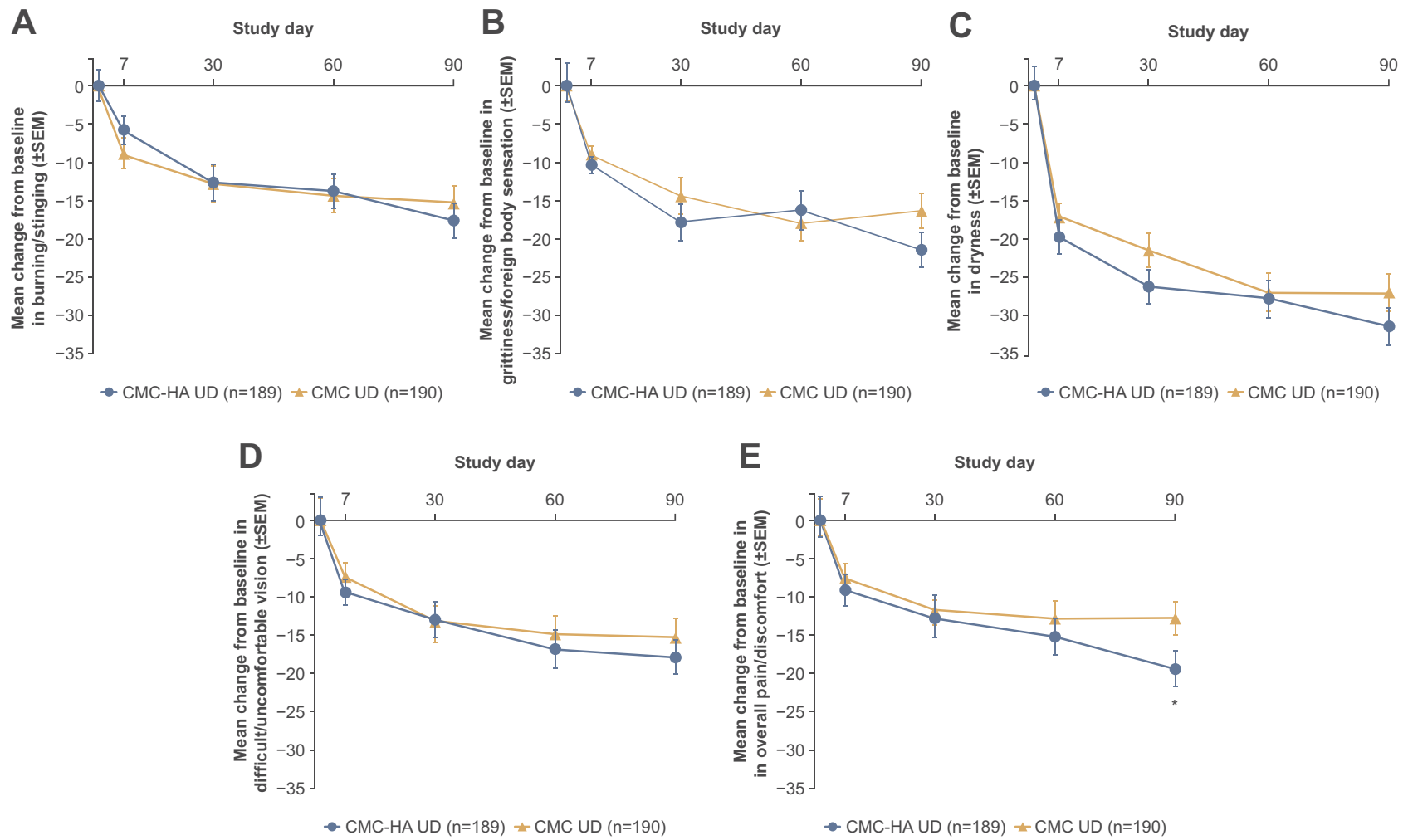

E

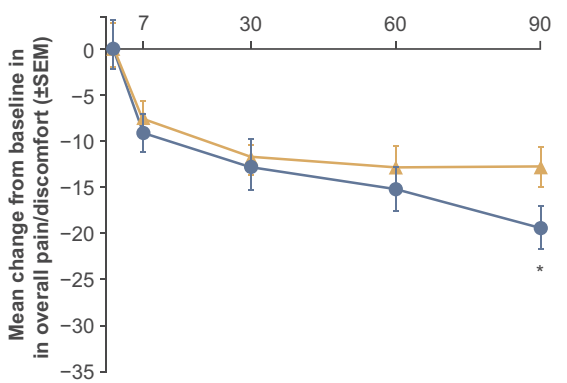

- CMC-HA UD $(n=189) \neq$ CMC UD $(n=190)$

Figure 3 Mean change in dry eye symptom scores from baseline at all study visits: (A) burning/stinging, (B) grittiness/foreign body sensation, (C) dryness, (D) difficult/ uncomfortable vision, and (E) overall ocular pain/discomfort (per-protocol population). ${ }^{*} P=0.048$ for $C M C-H A$ UD versus CMC UD.

Abbreviations: CMC, carboxymethylcellulose; HA, hyaluronic acid; SEM, standard error of mean; UD, unit dose. 
Table 4 Adverse Events Summary (Safety Population)

\begin{tabular}{|l|l|l|}
\hline Adverse Events, $\mathbf{n}(\%)$ & $\begin{array}{l}\text { CMC-HA UD } \\
\text { (n=224) }\end{array}$ & $\begin{array}{l}\text { CMC UD } \\
\text { (n=236) }\end{array}$ \\
\hline $\begin{array}{l}\text { All treatment-emergent } \\
\text { adverse events }\end{array}$ & $113(50.4)$ & $108(45.8)$ \\
$\begin{array}{l}\text { Treatment-related adverse } \\
\text { events }\end{array}$ & $23(10.3)$ & $25(10.6)$ \\
$\begin{array}{l}\text { Eye irritation } \\
\text { Blurred vision }\end{array}$ & $7(3.1)$ & $2(0.8)$ \\
Foreign body sensation in & $5(2.2)$ & $7(3.0)$ \\
eyes & $5(2.2)$ & $1(0.4)$ \\
$\begin{array}{l}\text { Conjunctival hyperemia } \\
\text { Eye pain }\end{array}$ & $3(1.3)$ & $4(1.7)$ \\
Photophobia & $3(1.3)$ & $2(0.8)$ \\
Instillation site pain & $3(1.3)$ & $0(0.0)$ \\
\hline
\end{tabular}

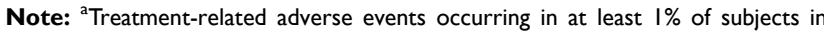
either treatment group listed below.

Abbreviations: CMC, carboxymethylcellulose; HA, hyaluronic acid; n, number of subjects; UD, unit dose.

and corneal staining at Days $7(P=0.02)$ and $30(P=0.01)$ in the aqueous-deficient cohort.

\section{Safety}

Within the safety population $(n=460)$, treatment-related adverse events were reported in $23(10.3 \%)$ subjects in the CMC-HA UD group and $25(10.6 \%)$ subjects in the CMC UD group; the most common treatment-related adverse events (experienced by at least $2 \%$ of subjects in either treatment group) were eye irritation, blurred vision, and foreign body sensation (Table 4). A total of 9 subjects (5 CMC-HA UD, 4 CMC UD) experienced serious adverse events, which were not considered related to treatment by the investigator, and none led to discontinuation from the study. A total of 24 subjects discontinued treatment due to adverse events, including 11 in the CMC-HA UD group and 13 in the CMC UD group.

Clinically significant biomicroscopy findings occurred with similar frequency in the CMC-HA UD (49 [21.9\%]) and CMC UD (51 [21.6\%]) treatment groups. Clinically significant biomicroscopy findings with an incidence rate $\geq 5 \%$ in either treatment group were conjunctival hyperemia, conjunctival edema, erythema of eyelid, and eyelid edema; the majority of findings were trace, mild, or moderate in severity. There were no significant differences between the two treatment groups in the frequency distribution of change from baseline in currently and bestcorrected visual acuity during the study; the majority of subjects in both treatment groups had "no change" in visual acuity at all follow-up visits. There were no clinically significant changes in IOP in either treatment group.

\section{Discussion}

In this randomized, double-masked study comparing two preservative-free $\mathrm{CMC}$-based artificial tears, treatment with both $\mathrm{CMC}-\mathrm{HA}$ and $\mathrm{CMC}$ alone led to significant improvement in signs and symptoms of dry eye from Day 7 onward. In the primary analysis, the CMC-HA UD formulation was non-inferior to CMC UD in terms of change in OSDI score from baseline to 90 days in the PP population, meeting the primary endpoint. Significant improvements from baseline in OSDI scores were observed with both CMC-HA UD and CMC UD at each study visit, but there was no significant difference between the formulations. Improvements in OSDI score were similar with both formulations irrespective of baseline symptom severity or baseline ocular staining.

Significant improvements in subjective measures of individual dry eye symptoms, subject acceptability and clinical signs of dry eye were also evident with both treatments in the secondary efficacy measures. A significant treatment difference in favor of CMC-HA UD was demonstrated in the Symptom Survey for overall ocular pain/discomfort at Day 90, and there was a trend in favor of CMC-HA UD at all timepoints for this category and those relating to difficult/uncomfortable vision and dryness. These findings were obtained at a dosing frequency of between 4.0 and 4.5 drops per day on average for both treatments.

Distinguishing between evaporative and aqueousdeficient dry eye is a critical factor in the successful management of DED. In this study, we identified evaporative dry eye subjects based on cut-offs described in the guidelines and previously reported studies, ${ }^{25,28-32}$ and demonstrated similar improvements with both formulations in signs and symptoms of dry eye irrespective of the subtype (evaporative, aqueous-deficient, or mixed dry eye), supporting the use of these treatments in a broad dry eye population. Significant benefits with CMC-HA UD were evident at earlier timepoints in terms of the composite score for ocular symptoms/vision-related function/ environmental triggers (ie, OSDI score) in the evaporative dry eye cohort, and in ocular pain/discomfort, difficult/ uncomfortable vision, and corneal staining in the aqueousdeficient cohort, in which there was also a general trend in favor of CMC-HA UD in the improvement of symptoms at Day 90. 
The study confirms a significant benefit of CMC-based artificial tear formulations in subjects with dry eye, who had used artificial tears prior to the study. The presence of the HA polymer appeared to confer additional advantages in terms of symptom relief, which was most evident in subjects with aqueous-deficient dry eye. The combination of $\mathrm{CMC}$ and $\mathrm{HA}$ has synergistic potential to further improve hydration and tolerability, as demonstrated by the decrease in ocular discomfort and pain in this group. The additional benefits seen with CMC-HA UD on symptom relief in aqueous-deficient dry eye may be attributed to the viscoelastic and hydrophilic properties of HA and its interaction with CMC. Despite sharing similar viscosity ( $15 \mathrm{cP}$; Table 1$)$, the rheological behavior of both formulations differs due to the interaction between CMC and HA. Combining both polymers promotes entanglement and increases the viscosity of the tear film, which in turn optimizes ocular hydration between blinks. Simmons et al demonstrated that the viscosity of a combined solution of $\mathrm{CMC}$ and $\mathrm{HA}$ was $60 \%$ higher than predicted by additive effects. ${ }^{21}$ In a rheological analysis of combinations of CMC and HA polymers at $0.5 \%+0.1 \%, 0.5 \%+0.15 \%$, and $1.0 \%+0.25 \%$, viscosity varied from $15.3 \mathrm{cP}$ (at $10 /$ second [open eye]) to $5.2 \mathrm{cP}$ (at 10,000/second [blinking eye]), $25.0 \mathrm{cP}$ (at 10/second) to $5.8 \mathrm{cP}$ (at 10,000/second), and $127.5 \mathrm{cP}$ (at 10/second) to $11.9 \mathrm{cP}$ (at $10,000 /$ second), respectively. ${ }^{21}$ During blinking, the polymer combination produced reduced viscosity (shear thinning), resulting in improved ocular comfort and reduced stickiness and blurring. ${ }^{21}$ Nevertheless, more quantitative data are warranted to further our understanding of this synergistic interaction. Other physicochemical differences between the formulations include the molecular weight of $\mathrm{CMC}$, which is low $(<100 \mathrm{kDa})$ in the CMC-HA formulation, versus a blend of medium $(\sim 250 \mathrm{kDa})$ and high $(\sim 700$ $\mathrm{kDa}$ ) in the CMC UD formulation, and the lactate versus borate buffer (Table 1); however, we would not expect the difference in buffers to impart any clinically relevant benefit. Both formulations are preservative-free and contain added organic osmolytes: glycerin, erythritol, and levocarnitine. While it is important to recognize the synergistic benefit of osmoprotectants used in conjunction with CMCand HA-based formulations, ${ }^{17}$ because both formulations in this study contained the same osmolytes, the most likely factors responsible for the observed benefit with CMC-HA relate to formulation differences in viscoelasticity and enhanced ocular surface protection via the CD-44 receptor, which binds $\mathrm{HA},{ }^{20}$ promoting ocular surface hydration and tear film stability.

The results reported in this study are consistent with previous studies investigating CMC- and HA-based formulations. Single polymer artificial tears containing CMC or HA alone have demonstrated comparable efficacy and safety. ${ }^{33-36}$ Previously, we demonstrated that a preserved formulation containing $\mathrm{CMC}$ and $\mathrm{HA}$ was non-inferior compared with a preserved formula containing CMC alone, and was efficacious in improving ocular symptoms and reducing ocular staining in a heterogeneous population of subjects with dry eye. ${ }^{18}$ The same preserved formulation was also reported to be non-inferior to a nonpreserved HA-based formula, with some advantages observed in symptom relief and subject acceptance. ${ }^{37}$ Formulations (with and without preservatives) combining CMC and HA have also demonstrated symptom reduction and improved clinical outcomes in contact lens wearers ${ }^{38}$ and following cataract ${ }^{39}$ or refractive surgery, ${ }^{40}$ which are both known to exacerbate dry eye symptoms. This postsurgical benefit with CMC and HA combined probably also relates to the additional mechanism of action involving activation of the $\mathrm{CD}-44$ receptor by $\mathrm{HA},{ }^{20}$ which helps promote corneal epithelial cell adhesion ${ }^{41}$ and migration, ${ }^{42}$ and improve ocular surface health. It is also important to consider those additional factors that can affect the behavior and physical property of HA in artificial tear formulations, such as the molecular weight of HA and the sodium concentration. High molecular weight HA, such as that used in the CMC-HA formulation in this study (ie, 1,178 kDa), allows higher low-shear viscosity at a lower concentration of HA. ${ }^{43,44}$ Furthermore, the molecular weight of HA is known to influence the production of inflammatory mediators; high molecular weight HA exhibits anti-inflammatory properties while low molecular weight HA promotes pro-inflammatory mediators. ${ }^{45}$ A low sodium concentration also appears to increase the waterbinding capacity of HA-containing formulations. ${ }^{46}$

Both formulations were well tolerated and the incidence of blurred vision, indicative of excessive viscosity, was comparable between CMC-HA UD and CMC UD.

Despite enrolling a heterogeneous dry eye population, the small cohort of aqueous-deficient dry eye subjects, in which a potential benefit with CMC-HA UD was evident, allows only preliminary conclusions to be made at this stage. Larger studies in specific subpopulations stratified by severity and etiology of dry eye are warranted to 
confirm whether the benefits observed in this study are applicable to the general dry eye population.

In conclusion, CMC-HA UD was shown to be noninferior to CMC UD in terms of symptom improvement after 3 months' treatment, meeting the primary endpoint of this study. Overall, this study demonstrates that the CMCHA UD artificial tear formulation is comparable with CMC UD in reducing signs and symptoms of DED; however, symptom improvement was generally more rapid and sustained with CMC-HA UD compared with CMC UD. Both formulations were effective regardless of the etiology of dry eye, although there was evidence to suggest a potential benefit with CMC-HA UD versus CMC UD in subjects with aqueous-deficient dry eye. The CMC-HA UD formulation was safe and well tolerated, with a similar safety profile to CMC UD. These results support the use of this non-preserved CMC-HA UD formulation in a broad range of subjects with dry eye.

\section{Data Sharing Statement}

Allergan (an AbbVie company) will share de-identified subject-level data and study-level data including protocols and clinical study reports for phase 2, 3 or 4 trials completed after 2008 that are registered to ClinicalTrials.gov or EudraCT, have received regulatory approval in the United States and/or the European Union in a given indication and the primary manuscript from the trial has been published. To request access to the data, the researcher must sign a data use agreement and any shared data is to be used for non-commercial purposes. More information can be found on http://www.allerganclinicaltrials.com/.

\section{Acknowledgments}

This study was sponsored by Allergan plc, Dublin, Ireland (prior to acquisition by AbbVie Inc.). Writing and editorial assistance was provided to the authors by Kakuri Omari, $\mathrm{PhD}$, CMPP, and Stuart Murray, MSc, CMPP of Evidence Scientific Solutions, Inc, Philadelphia, PA, and funded by AbbVie Inc. All authors met the ICMJE authorship criteria. Neither honoraria nor payments were made for authorship. The following principal investigators participated in this study: Dr. Parwez Hossain, Southampton, UK; Aravind Reddy, Aberdeen, UK; Prof. Francisco Figueiredo, Newcastle-upon-Tyne, UK; Subhanjan Mukherji, Great Yarmouth, UK; Dr. Ejaz Ansari, Maidstone, UK; Prof. Algis Vingrys, Melbourne, Australia; Dr. Geoffrey Cohn, Sydney, Australia; Dr. Michael A. Coote, Melbourne, Australia; Dr. Mark D. Daniell, Melbourne, Australia; Prof. Minas T. Coroneo, Sydney, Australia;
Dr. Jacqueline Tan, Sydney, Australia; Dr. Renuka Bathija, Melbourne, Australia; Prof. Ingeborg Stalmans, Leuven, Belgium; Dr. Laurence Postelmans, Brussels, Belgium; Dr. Christina P. Martinez, Valencia, Spain; Dr. Maite S. de la Maza Serra, Barcelona, Spain; Dr. Jesus M. Iruzubieta, Sevilla, Spain; Prof. Jose M. Benitez-del-Castillo, Madrid, Spain; Dr. Margarita C. Cano, Valladolid, Spain; Dr. Rafael G. Pina, Huelva, Spain; Dr. Sergio P. Sueiro, Vizcaya, Spain; Dr. Stephan Fauquier, Marseille, France; Prof. Pierre-Jean Pisella, Tours, France; Prof. Frederic Chiambaretta, ClermontFerrand, France; Prof. Dominique Bremond-Gignac, Amiens, France; Prof. Beatrice Cochener, Brest, France; Dr. Cedric Schweitzer, Bordeaux, France; Dr. Catherine CreuzotGarcher, Dijon, France; Prof. Daniel Boehringer, Freiburg, Germany; Dr. Katrin Lorenz, Mainz, Germany; Dr. Phillip Steven, Cologne, Germany; Dr. Elisabeth Messmer, Munich, Germany; Dr. Andrei Nestler, Leipzig, Germany; Dr. Jorg M. Koch, Munster, Germany; Prof Berthold Seitz, Homburg Saar, Germany; Dr. Christina Jacobi, Erlangen, Germany; Prof. Andrea Leonardi, Padova, Italy; Dr. Giulio M. Modorati, Milan, Italy; Prof. Marco Nardi, Pisa, Italy; Prof. Ugo Menchini, Firenze, Italy; Prof. Gabriella J. Orsoni, Parma, Italy; Prof. Pasquale Aragona, Messina, Italy; Prof. Yury Astakhov, St. Petersburg, Russia; Prof. Vladimir Brzheskiy, St. Petersburg, Russia; Prof. Valeriy Erichev, Moscow, Russia.

\section{Disclosure}

P Aragona has received consultancy fees, research grants and/or speaker fees from Allergan (an AbbVie company), Alcon Italia, SIFI, Medivis, Dompé, OFF, Novartis, Shire, Santen, and Thea. JM Benítez-del-Castillo has received personal fees from Allergan (an AbbVie company), Alcon-Novartis, Thea, Santen, Bausch+Lomb, Dompé, Visufarma, Angelini and Brill. MT Coroneo reports royalties and/or personal fees from Alcon, Allgenesis, DORC, Insight Surgical, Katena, and Novartis/Shire, outside the submitted work. S Mukherji has received non-financial support from Allergan (an AbbVie company) and Alcon-Novartis. $J$ Tan has received research grants from Alcon, Allergan (an AbbVie company), Azura Ophthalmics, Stiltec, CooperVision, Menicon, nthalmic, and Kedalion. A Vingrys has received research grants from Alcon, Allergan (an AbbVie company), and CooperVision. $\mathrm{H}$ Liu and C Carlisle-Wilcox are employees of AbbVie Inc. PA Simmons and $J$ Vehige were employees of Allergan plc, Irvine, CA, USA, when the study was 
conducted. PA Simmons reports personal fees from Allergan, during the conduct of the study; personal fees from Allergan plc, outside the submitted work. In addition, PA Simmons and J Vehige have a patent US 8569367 issued to Allergan plc, and a patent US 8569370 issued to Allergan plc. PA Simmons, J Vehige, and H Liu have a patent US 2014/0221309 A1 pending to Allergan plc. The authors report no other conflicts of interest in this work.

\section{References}

1. Craig JP, Nichols KK, Akpek EK, et al. TFOS DEWS II definition and classification report. Ocul Surf. 2017;15(3):276-283. doi:10.1016/j.jtos.2017.05.008

2. Stapleton F, Alves M, Bunya VY, et al. TFOS DEWS II epidemiology report. Ocul Surf. 2017;15(3):334-365. doi:10.1016/j. jtos.2017.05.003

3. Mertzanis P, Abetz L, Rajagopalan K, et al. The relative burden of dry eye in patients' lives: comparisons to a U.S. normative sample. Invest Ophthalmol Vis Sci. 2005;46(1):46-50. doi:10.1167/iovs.030915

4. Miljanović B, Dana R, Sullivan DA, Schaumberg DA. Impact of dry eye syndrome on vision-related quality of life. Am J Ophthalmol. 2007;143(3):409-415. doi:10.1016/j.ajo.2006.11.060

5. Behrens A, Doyle JJ, Stern L, et al. Dysfunctional tear syndrome: a Delphi approach to treatment recommendations. Cornea. 2006;25 (8):900-907. doi:10.1097/01.ico.0000214802.40313.fa

6. Jones L, Downie LE, Korb D, et al. TFOS DEWS II management and therapy report. Ocul Surf. 2017;15(3):575-628. doi:10.1016/j. jtos.2017.05.006

7. Baudouin C, Aragona P, Messmer EM, et al. Role of hyperosmolarity in the pathogenesis and management of dry eye disease: proceedings of the OCEAN group meeting. Ocul Surf. 2013;11(4):246-258. doi:10.1016/j.jtos.2013.07.003

8. Stahl U, Willcox M, Stapleton F. Osmolality and tear film dynamics. Clin Exp Optom. 2012;95(1):3-11. doi:10.1111/j.1444-0938.2011.00634.x

9. Pflugfelder SC, Corrales RM, de Paiva CS. T helper cytokines in dry eye disease. Exp Eye Res. 2013;117:118-125. doi:10.1016/j. exer.2013.08.013

10. Stevenson W, Chauhan SK, Dana R. Dry eye disease: an immune-mediated ocular surface disorder. Arch Ophthalmol. 2012;130(1):90-100. doi:10.1001/archophthalmol.2011.364

11. Hua X, Deng R, Li J, et al. Protective effects of L-carnitine against oxidative injury by hyperosmolarity in human corneal epithelial cells. Invest Ophthalmol Vis Sci. 2015;56(9):5503-5511. doi:10.1167/ iovs.14-16247

12. Khandekar N, Willcox MD, Shih S, Simmons P, Vehige J, Garrett Q. Decrease in hyperosmotic stress-induced corneal epithelial cell apoptosis by L-carnitine. Mol Vis. 2013;19:1945-1956.

13. Hua X, Su Z, Deng R, Lin J, Li DQ, Pflugfelder SC. Effects of L-carnitine, erythritol and betaine on pro-inflammatory markers in primary human corneal epithelial cells exposed to hyperosmotic stress. Curr Eye Res. 2015;40(7):657-667. doi:10.3109/0271368 3.2014.957776

14. Giannaccare G, Fresina M, Versura P. A novel osmoprotectant tear substitute for the treatment of dry eye disease. Int J Ophthalmol Clin Res. 2016;3(3):058. doi:10.23937/2378-346X/1410058

15. Brocker C, Thompson DC, Vasiliou V. The role of hyperosmotic stress in inflammation and disease. Biomol Concepts. 2012;3 (4):345-364. doi:10.1515/bmc-2012-0001
16. Tampucci S, Monti D, Burgalassi S, et al. Effect of 5-oxo-2-pyrrolidinecarboxylic acid (PCA) as a new topically applied agent for dry eye syndrome treatment. Pharmaceutics. 2018;10(3):137. doi:10.33 90/pharmaceutics 10030137

17. Mateo Orobia AJ, Saa J, Ollero Lorenzo A, Herreras JM. Combination of hyaluronic acid, carmellose, and osmoprotectants for the treatment of dry eye disease. Clin Ophthalmol. 2018;12:453-461. doi:10.2147/opth.S157853

18. Simmons PA, Liu H, Carlisle-Wilcox C, Vehige JG. Efficacy and safety of two new formulations of artificial tears in subjects with dry eye disease: a 3-month, multicenter, active-controlled, randomized trial. Clin Ophthalmol. 2015;9:665-675. doi:10.2147/OPTH. S78184

19. Garrett Q, Simmons PA, Xu S, et al. Carboxymethylcellulose binds to human corneal epithelial cells and is a modulator of corneal epithelial wound healing. Invest Ophthalmol Vis Sci. 2007;48(4):1559-1567. doi:10.1167/iovs.06-0848

20. Lerner LE, Schwartz DM, Hwang DG, Howes EL, Stern R. Hyaluronan and CD44 in the human cornea and limbal conjunctiva. Exp Eye Res. 1998;67(4):481-484. doi:10.1006/ exer.1998.0567

21. Simmons PA, Vehige JG. Investigating the potential benefits of a new artificial tear formulation combining two polymers. Clin Ophthalmol. 2017;11:1637-1642. doi:10.2147/OPTH.S135550

22. Chen W, Zhang X, Li J, et al. Efficacy of osmoprotectants on prevention and treatment of murine dry eye. Invest Ophthalmol Vis Sci. 2013;54(9):6287-6297. doi:10.1167/iovs.13-12081

23. Corrales RM, Luo L, Chang EY, Pflugfelder SC. Effects of osmoprotectants on hyperosmolar stress in cultured human corneal epithelial cells. Cornea. 2008;27(5):574-579. doi:10.1097/ICO.0b013e31 $8165 \mathrm{~b} 19 \mathrm{e}$

24. Schiffman RM, Christianson MD, Jacobsen G, Hirsch JD, Reis BL. Reliability and validity of the ocular surface disease index. Arch Ophthalmol. 2000;118(5):615-621. doi:10.1001/ archopht.118.5.615

25. Wolffsohn JS, Arita R, Chalmers R, et al. TFOS DEWS II diagnostic methodology report. Ocul Surf. 2017;15(3):539-574. doi:10.1016/j. jtos.2017.05.001

26. Aragona P, Cannavò SP, Borgia F, Guarneri F. Utility of studying the ocular surface in patients with acne vulgaris treated with oral isotretinoin: a randomized controlled trial. Br J Dermatol. 2005;152 (3):576-578. doi:10.1111/j.1365-2133.2005.06389.x

27. Morikawa T, Yoshida M. A useful testing strategy in phase III trials: combined test of superiority and test of equivalence. J Biopharm Stat. 1995;5(3):297-306. doi:10.1080/10543409508835115

28. Pflugfelder SC, Tseng SC, Sanabria O, et al. Evaluation of subjective assessments and objective diagnostic tests for diagnosing tear-film disorders known to cause ocular irritation. Cornea. 1998;17 (1):38-56. doi:10.1097/00003226-199801000-00007

29. Rolando M, Valente C, Barabino S. New test to quantify lipid layer behavior in healthy subjects and patients with keratoconjunctivitis sicca. Cornea. 2008;27(8):866-870. doi:10.1097/ICO.0b013e318 $16 \mathrm{f} 630 \mathrm{e}$

30. van Bijsterveld OP. Diagnostic tests in the Sicca syndrome. Arch Ophthalmol. 1969;82(1):10-14. doi:10.1001/archopht.1969.0099002 0012003

31. Pflugfelder SC, Tseng SC, Yoshino K, Monroy D, Felix C, Reis BL. Correlation of goblet cell density and mucosal epithelial membrane mucin expression with rose Bengal staining in patients with ocular irritation. Ophthalmology. 1997;104(2):223-235. doi:10.1016/S01616420(97)30330-3

32. Abelson MB, Ousler GW, Nally LA, Welch D, Krenzer K. Alternative reference values for tear film break up time in normal and dry eye populations. Adv Exp Med Biol. 2002;506(Pt B):1121-1125. 
33. Lee JH, Ahn HS, Kim EK, Kim TI. Efficacy of sodium hyaluronate and carboxymethylcellulose in treating mild to moderate dry eye disease. Cornea. 2011;30(2):175-179. doi:10.1097/ICO.0b013e318 1e9adcc

34. Baudouin C, Cochener B, Pisella PJ, et al. Randomized, phase III study comparing osmoprotective carboxymethylcellulose with sodium hyaluronate in dry eye disease. Eur J Ophthalmol. 2012;22 (5):751-761. doi:10.5301/ejo.5000117

35. Aragona P, Di Stefano G, Ferreri F, Spinella R, Stilo A. Sodium hyaluronate eye drops of different osmolarity for the treatment of dry eye in Sjögren's syndrome patients. $\mathrm{Br} J$ Ophthalmol. 2002;86 (8):879-884. doi:10.1136/bjo.86.8.879

36. Aragona P, Papa V, Micali A, Santocono M, Milazzo G. Long term treatment with sodium hyaluronate-containing artificial tears reduces ocular surface damage in patients with dry eye. $\mathrm{Br} J$ Ophthalmol. 2002;86(2):181-184. doi:10.1136/bjo.86.2.181

37. Labetoulle M, Chiambaretta F, Shirlaw A, Leaback R, Baudouin C. Osmoprotectants, carboxymethylcellulose and hyaluronic acid multi-ingredient eye drop: a randomised controlled trial in moderate to severe dry eye. Eye. 2017;31(10):1409-1416. doi:10.1038/ eye. 2017.73

38. Nichols JJ, Lievens CW, Bloomenstein MR, Liu H, Simmons P, Vehige J. Dual-polymer drops, contact lens comfort, and lid wiper epitheliopathy. Optom Vis Sci. 2016;93(8):979-986. doi:10.1097/ OPX.0000000000000878

39. Mencucci R, Boccalini C, Caputo R, Favuzza E. Effect of a hyaluronic acid and carboxymethylcellulose ophthalmic solution on ocular comfort and tear-film instability after cataract surgery. $J$ Cataract Refract Surg. 2015;41(8):1699-1704. doi:10.1016/j. jcrs.2014.12.056
40. Wallerstein A, Jackson WB, Chambers J, Moezzi A, Lin H, Simmons PA. Management of post-LASIK dry eye: a multicenter randomized comparison of a new multi-ingredient artificial tear to carboxymethylcellulose. Clin Ophthalmol. 2018;12:839. doi:10.2147/ OPTH.S163744

41. Nishida T, Nakamura M, Mishima H, Otori T. Hyaluronan stimulates corneal epithelial migration. Exp Eye Res. 1991;53(6):753-758. doi:10.1016/0014-4835(91)90110-Z

42. Inoue M, Katakami C. The effect of hyaluronic acid on corneal epithelial cell proliferation. Invest Ophthalmol Vis Sci. 1993;34 (7):2313-2315.

43. Aragona P, Simmons PA, Wang H, Wang T. Physicochemical properties of hyaluronic acid-based lubricant eye drops. Transl Vis Sci Tech. 2019;8(6):2. doi:10.1167/tvst.8.6.2

44. Rivas M, Aragona P, Simmons P, Wang H, Vehige J. Physical properties of hyaluronic acid-based eye drops predict viscosity and potential clinical performance. Presented as a poster at: Controversies in Ophthalmology (COPHy) - 8th World Congress; March 30, 2017; Madrid, Spain.

45. Rayahin JE, Buhrman JS, Zhang Y, Koh TJ, Gemeinhart RA. High and low molecular weight hyaluronic acid differentially influence macrophage activation. ACS Biomater Sci Eng. 2015;1(7):481-493. doi:10.1021/acsbiomaterials.5b00181

46. Simmons P, Wang H, Wang T, Meller R, Vehige J. Sodium concentration affects the physical properties of hyaluronic acid-based lubricant eye drops. Invest Ophthalmol Vis Sci. 2018;59:4899.
Clinical Ophthalmology

\section{Publish your work in this journal}

Clinical Ophthalmology is an international, peer-reviewed journal covering all subspecialties within ophthalmology. Key topics include: Optometry; Visual science; Pharmacology and drug therapy in eye diseases; Basic Sciences; Primary and Secondary eye care; Patient Safety and Quality of Care Improvements. This journal is indexed on PubMed
Dovepress

Central and CAS, and is the official journal of The Society of Clinical Ophthalmology (SCO). The manuscript management system is completely online and includes a very quick and fair peer-review system, which is all easy to use. Visit http://www.dovepress.com/ testimonials.php to read real quotes from published authors. 